\title{
Evidence for Spinodal Decomposition in Nuclear Multifragmentation
}

\author{
B. Borderie ${ }^{1}$, G. Tăbăcaru ${ }^{1,2}$, Ph. Chomaz ${ }^{3}$, M. Colonna ${ }^{4}$, A. Guarnera ${ }^{4}$, M. Pârlog ${ }^{2}$, M.F Rivet $^{1}$ \\ and \\ G. Auger ${ }^{3}$, Ch. O. Bacri ${ }^{1}$, N. Bellaize ${ }^{5}$, R. Bougault ${ }^{5}$, B. Bouriquet ${ }^{3}$, R. Brou ${ }^{5}$, P. Buchet ${ }^{6}$, A. Chbihi $^{3}$, J. Colin $^{5}$, \\ A. Demeyer ${ }^{7}$, E. Galichet ${ }^{1,8}$, E. Gerlic ${ }^{7}$, D. Guinet ${ }^{7}$, S. Hudan ${ }^{3}$, P. Lautesse ${ }^{7}$, F. Lavaud ${ }^{1}$, J.L. Laville ${ }^{3}$, \\ J.F. Lecolley ${ }^{5}$, C. Leduc ${ }^{7}$, R. Legrain ${ }^{6}$, N. Le Neindre ${ }^{5}$, O. Lopez ${ }^{5}$, M. Louvel ${ }^{5}$, A.M. Maskay ${ }^{7}$, J. Normand ${ }^{5}$, \\ P. Pawłowski ${ }^{1}$, E. Rosato ${ }^{9}$, F. Saint-Laurent ${ }^{3}$, J.C. Steckmeyer ${ }^{5}$, B. Tamain $^{5}$, L. Tassan-Got ${ }^{1}$, E. Vient ${ }^{5}$, \\ J.P. Wieleczko ${ }^{3}$ \\ INDRA Collaboration \\ 1 Institut de Physique Nucléaire, IN2P3-CNRS, F-91406 Orsay Cedex, France. \\ ${ }^{2}$ National Institute for Physics and Nuclear Engineering, RO-76900 Bucharest-Măgurele, Romania. \\ ${ }^{3}$ GANIL, CEA et IN2P3-CNRS, B.P. 5027, F-14076 Caen Cedex, France. \\ ${ }^{4}$ Laboratorio Nazionale del Sud, Viale Andrea Doria, I-95129 Catania, Italy. \\ ${ }^{5}$ LPC, IN2P3-CNRS, ISMRA et Université, F-14050 Caen Cedex, France. \\ ${ }^{6}$ DAPNIA/SPhN, CEA/Saclay, F-91191 Gif sur Yvette Cedex, France. \\ 7 Institut de Physique Nucléaire, IN2P3-CNRS et Université, F-69622 Villeurbanne Cedex, France. \\ 8 Conservatoire National des Arts et Métiers, F75141 Paris cedex 03. \\ 9 Dipartimento di Scienze Fisiche e Sezione INFN, Università di Napoli "Federico II", I80126 Napoli, Italy.
}

(August 19, 2019)

Multifragmentation of a "fused system" was observed for central collisions between $32 \mathrm{MeV} /$ nucleon ${ }^{129} \mathrm{Xe}$ and ${ }^{\text {nat }} \mathrm{Sn}$. Most of the resulting charged products were well identified thanks to the high performances of the INDRA $4 \pi$ array. Experimental higher-order charge correlations for fragments show a weak but non ambiguous enhancement of events with nearly equal-sized fragments. Supported by dynamical calculations in which spinodal decomposition is simulated, this observed enhancement is interpreted as a "fossil" signal of spinodal instabilities in finite nuclear systems.

25.70.-z, 25.70.Pq, 24.60.Ky

The decay of highly excited nuclear systems through multifragmentation is, at present time, a subject of great interest in nucleus-nucleus collisions, and many efforts are made to understand the underlying physics. While this process has been observed for many years, its experimental knowledge was strongly improved only recently with the advent of powerful $4 \pi$ devices which authorize careful selections of well defined fused systems undergoing multifragmentation. Many theories have been developed to explain multifragmentation (see for example ref. [1] for a general review of models). One theory, in particular, is the concept of multifragmentation which considers volume instabilities of the spinodal type. Indeed, during a collision, a wide zone of the nuclear matter phase diagram may be explored and the nuclear system may enter the liquid-gas phase coexistence region (at low density) and even more precisely the unstable spinodal region (domain of negative incompressibility) 22. Thus, a possible origin of multifragmentation may be found through the growth of density fluctuations in this unstable region. Within this theoretical scenario a breakup into nearly equal-sized "primitive" fragments should be favored in relation with the wave-lengths of the most unstable modes present in the spinodal region [3]. However this simple picture is expected to be strongly blurred by several effects: the beating of different modes, eventual coalescence of nascent fragments, secondary decay of excited fragments and mainly the finite size of the system [4]. Therefore only a weak proportion of multifragmentation events with nearly equal-sized fragments is expected. Note that surface instabilities developed from peculiar multifragmenting geometrical structures (bubbles, disk-like), can also produce such events 佂. In this letter we investigate, using a very sensitive method of charge correlations, the occurrence of equal-sized fragment partitions in a selected sample of experimental events, corresponding to a fused system undergoing multifragmentation. A comparison is made with predictions of $3 \mathrm{D}$ stochastic mean-field simulations of collisions which take into account the dynamics of the most unstable modes in the spinodal region.

We have experimentally studied the reaction ${ }^{129} \mathrm{Xe}+{ }^{\text {nat }} \mathrm{Sn}$ at $32 \mathrm{MeV} /$ nucleon. The measurements were performed at the GANIL accelerator using the $4 \pi$ multidetector for charged reaction products INDRA [5]. Detailed information on the experiment and on the selection of fused events can be found in Ref. [6, [7]. Let us just recall that the selection was performed by requiring the detection of a significant fraction $(\geq 80 \%)$ of the charge of the system; these selected events are called complete events. Reaction products with charge $\mathrm{Z} \geq 5$ were defined as fragments. Finally the preferred direction of emission of matter in the center of mass of the reaction (flow angle) was determined from the calculation of the energy tensor of fragments, and the require- 
ment was made that this angle be larger than $60^{\circ}$ [8,7]. The main argument underlying the chosen selection is that while a fused system should be present at all flow angles, binary dissipative collisions should vanish when this angle is large, giving way to an almost pure "fusion" phenomenon [9]. The present selection corresponds to $\sim 25$ $\mathrm{mb}$, and the total cross-section for multifragmentation of compact shape systems is estimated to $100 \mathrm{mb}$. Figure 1 exhibits how complete events populate the flow angle domain as a function of their measured total kinetic energy (i.e. c.m. kinetic energy of all detected fragments and light charged particles).

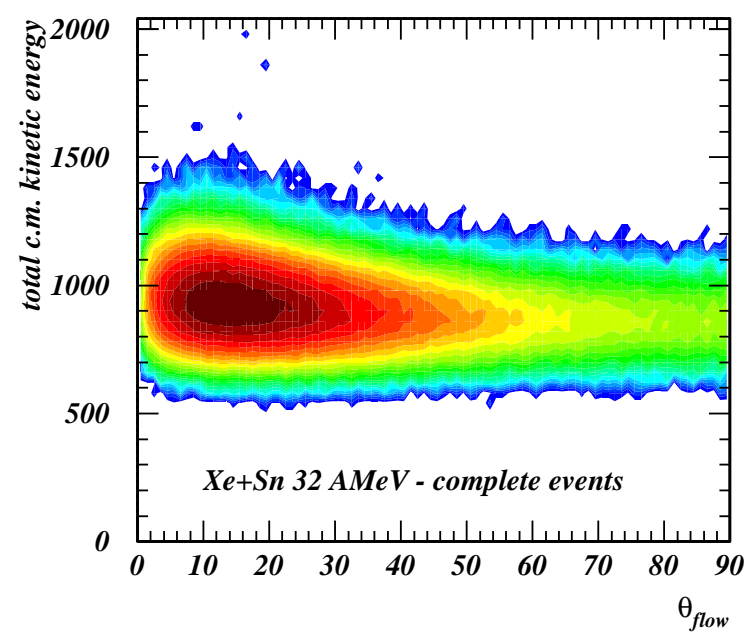

FIG. 1. Wilczynski diagram for complete events: correlation between total measured c.m. kinetic energy and flow angle $\theta_{\text {flow }}$.

An unambiguous charge identification is essential for building unbiased charge correlation functions: in this experiment all fragments with charge $\mathrm{Z} \leq 20$ are identified with a resolution of one charge unit. The correlation method used, called "higher order correlations" was proposed a few years ago in ref [10. It is appropriate to search for weak signals and its originality consists of the fact that all information on fragments of one event is condensed in two variables to construct the charge correlation. This quantity is defined by the expression:

$$
\left.\frac{Y(\Delta Z,<Z>)}{Y^{\prime}(\Delta Z,<Z>)}\right|_{M}
$$

Here, $Y(\Delta Z,<Z>)$ is the yield of selected events with $<Z>$ and $\Delta Z$ values; $M$ is the fragment multiplicity, $<Z>$ denotes the average fragment charge of the event:

$$
<>=\frac{1}{M} \sum_{i=1}^{M} Z_{i}
$$

and $\Delta Z$ is the standard deviation, defined by:

$$
\Delta Z=\sqrt{\frac{1}{M-1} \sum_{i=1}^{M}\left(Z_{i}-<Z>\right)^{2}}
$$

The denominator $Y^{\prime}(\Delta Z,<Z>)$ which represents the uncorrelated yield is built, for each fragment multiplicity, by taking fragments in different events of the selected sample. The number of uncorrelated events is chosen large enough $\left(10^{3}\right.$ per true event) to strongly reduce their contribution to statistical error. With such a correlation method, if events with nearly equal-sized fragments are produced, we expect to see peaks appearing in the first $\Delta \mathrm{Z}$ bin $(0-1)$.

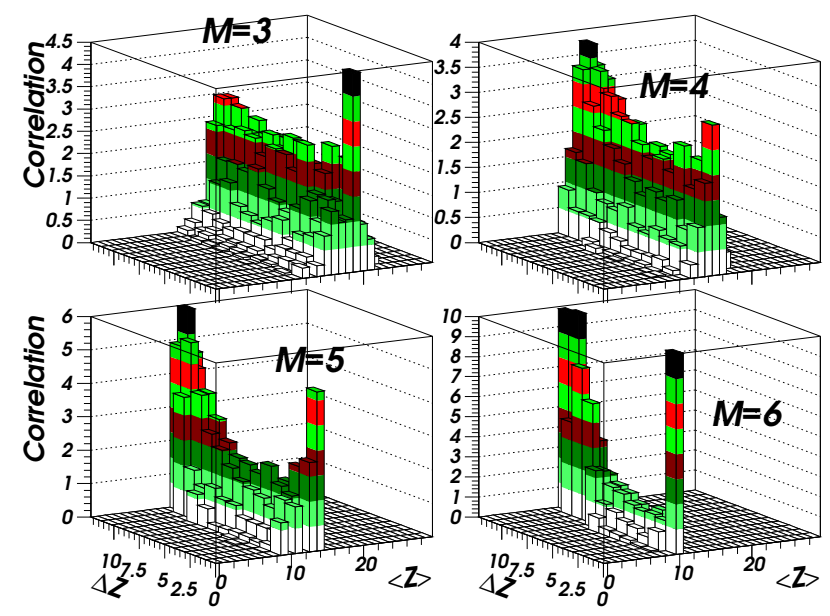

FIG. 2. Experimental higher-order charge correlations for selected fusion events in $32 \mathrm{MeV} /$ nucleon $\mathrm{Xe}+\mathrm{Sn}$ collisions.

Higher order correlation functions for selected experimental events are shown in fig. 2. We observe peaks in the bin $\Delta \mathrm{Z}=0-1$ for each fragment multiplicity. From $\mathrm{M}=3$ to $\mathrm{M}=6$ the maximum is moving from $\langle Z\rangle=19$ to $\langle Z>=10$. When multiplied by the multiplicity these lead to a roughly constant value close to the most probable total fragment charge for all fused events. It was moreover verified that the kinetic energy of fragments in these events does not differ (within statistical error) from average kinetic energies of fragments for other selected events.

We can now estimate whether the enhancement of events with equal-sized fragments is statistically significant and quantify their occurrence. In this aim we built charge correlations for all events, whatever their multiplicity, by replacing the variable $<Z>$ by $Z_{\text {tot }}=$ $\mathrm{M} \times<Z>$. For this compact presentation uncorrelated events are built and weighted in proportion to real events of each multiplicity. For each bin in $Z_{t o t}$, fixed at six atomic number units, an exponential evolution of the correlation function is observed from $\Delta \mathrm{Z}=7-8$ down to $\Delta \mathrm{Z}=2-3$. This exponential evolution is thus taken as "background" to extrapolate down to the first $\Delta \mathrm{Z}$ bin. Higher order correlation functions for the first bin in $\Delta \mathrm{Z}$ 
are displayed in Fig. 3 with their statistical errors; the solid line corresponds to the extrapolated "background". All events corresponding to the three points whose error bar is fully located above this line correspond to a statistically significant enhancement of equal-sized fragment partitions. The probabilities that these values which are higher than the background simply arise from statistical fluctuations are $0.048\left(Z_{t o t}=45\right), 0.038\left(Z_{t o t}=51\right)$ and $0.022\left(Z_{t o t}=57\right)$. The number of significant events amounts to $0.1 \%$ of selected fusion events.

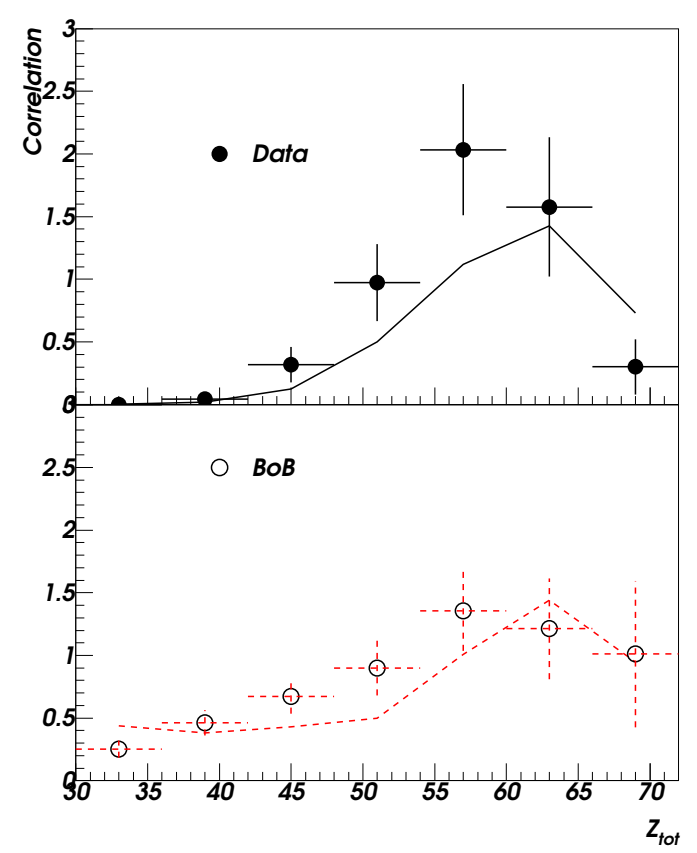

FIG. 3. Higher-order charge correlations: quantitative results for experimental data (upper panel) and simulations (lower panel). Symbols indicate the events where $\Delta \mathrm{Z}=0-1$, curves show the background defined extrapolating $\Delta \mathrm{Z}>2$ (see text). Vertical bars correspond to statistical errors assuming independent measurements and horizontal bars define $Z_{\text {tot }}$ bins.

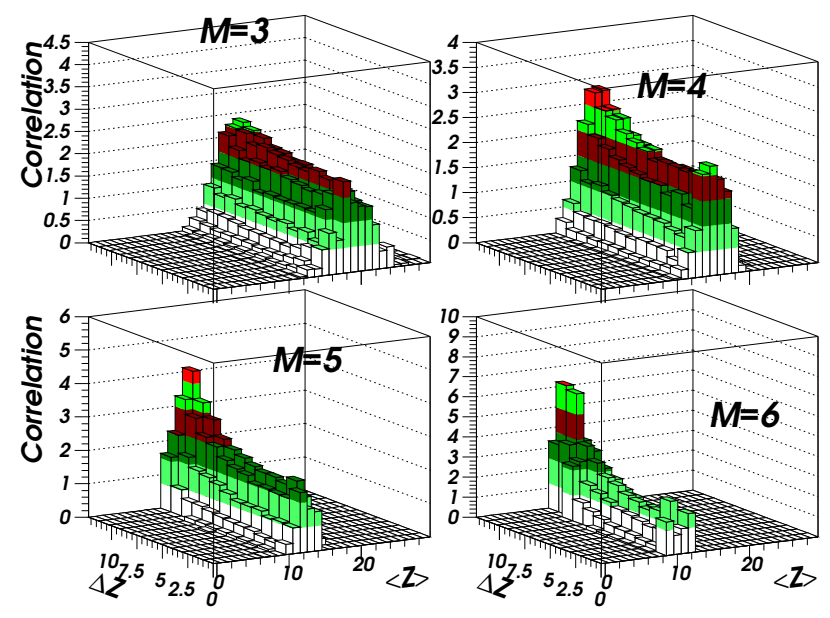

FIG. 4. Experimental higher-order charge correlations for very dissipative "binary" collisions in $32 \mathrm{MeV} /$ nucleon Xe+Sn collisions (see text).

To test if these equal-sized fragment partitions were specific - or not - of the selected fused events, we have studied a data sample dominated by very dissipative collisions of binary type. This is done by selecting in the Wilczynsky diagram for complete events (see fig. 11) total c.m. energy lower than $1200 \mathrm{MeV}$ with a flow angle $\theta_{\text {flow }} \leq 30^{\circ}$. Within this new selection we estimate that about $9 \times 10^{3}$ fused events are mixed with about $3.6 \times 10^{5}$ binary (or eventually strongly deformed, or both) events. The correlation functions are presented in fig. 4. No statistically significant signals are observed. The small peak for $M=6$ corresponds to $0.0008 \%$ of selected events. This is an indication that only fused events are characterized by an enhanced production of nearly equal-sized fragments.

In ref. [6], it was experimentally observed that charge distributions for fusion/multifragmentation events were independent of the total mass of the incident nuclei (248 or $393 \mathrm{u}$ ), while fragment multiplicities scaled as their total charge. These properties were the first hint of a bulk effect for producing fragments.

Dynamical stochastic mean-field simulations [11, 12 were performed for head-on collisions only, thus neglecting shape effects. Spinodal decomposition is simulated using the Brownian one-Body (BoB) dynamics [13 15], which consists in employing a Brownian force in the kinetic equations. The ingredients of the simulations are as follows. The self-consistent mean field potential |16 chosen gives a soft equation of state $\left(\mathrm{K}_{\infty}=200 \mathrm{MeV}\right)$ and the finite range of the nuclear interaction is taken into account using a convolution with a gaussian function with a width of $0.9 \mathrm{fm}$ [17]. The addition of a term proportional to $\Delta \rho$ in the mean-field potential allows to wellreproduce the surface energy of ground-state nuclei [18]. This is essential in order to correctly describe the expansion dynamics of the fused system. In the collision term a constant $\sigma_{n n}$ value of $41 \mathrm{mb}$, without in-medium, energy, isospin or angle dependence is used [19]. In the following step the spatial configuration of the primary fragments, with their excitation energies as produced by BoB, was taken as input in the SIMON code [20] to follow the fragment deexcitation while preserving spacetime correlations. Finally the events were filtered to account for the experimental device. These complete simulations well reproduce multiplicity and charge distributions of fragments and their average kinetic energies [15]. To refine the comparison, higher-order charge correlations are calculated for the simulated events. Although all events in the simulation arise from spinodal decomposition, only a very small fraction, as suspected for finite systems [4], exhibits final partitions with equal-sized fragments. This fraction is not strongly modified by the 
de-excitation of fragments; as an example $\mathrm{Z}=15$ primary fragments produced in BoB simulations (with their mass and excitation energy distributions) lead to a secondary $\mathrm{Z}$ distribution centered at $\mathrm{Z}=14$ with a standard deviation of 0.6. Finally the proportion of statistically significant equal-sized fragment partitions is similar $(0.15 \%)$ to the experimental one. A detailed quantitative comparison is displayed in fig. 3. The similarities between experimental and calculated events allow to attribute all fusion-multifragmentation events to spinodal decomposition. The peaks observed near $\Delta Z=0$ in the higher-order charge correlations are thus fossil fingerprints of the partitions expected from spinodal decomposition. It was previously observed that multiplicity and $\mathrm{Z}$ distributions, and the average kinetic energy of fragments were also well reproduced with the statistical model SMM [21,22]. It is interesting to underline that in this sample of SMM events no partition with $\Delta Z<2$ exists. Note also that fragment velocity correlations [23] are perfectly reproduced either in BoB simulations, or in the breakup of systems with elongated shapes. As none of these configurations is strongly necked or close to a bubble, we can exclude surface instabilities as a possible source of equalsized fragments, in favor of bulk instabilities [6, 24, 25].

In conclusion, we have investigated charge correlation functions for fusion events which undergo multifragmentation. We have found a weak but unambiguously enhanced production of events with equal-sized fragments. Supported by theoretical simulations we interpret this enhancement as a signature of spinodal instabilities as the origin of multifragmentation in the Fermi energy domain. Spinodal instabilities are thus shown for the first time in a finite system. Moreover the occurrence of spinodal decomposition shows the presence of a liquid-gas coexistence region and gives a strong argument in favor of the existence of a first order liquid-gas phase transition in finite nuclear systems.

[1] L. G. Moretto and G. J. Wozniak, Ann. Rev. of Nuclear and Particle Science 43 (1993) 379 and references therein.

[2] G. F. Bertsch and P. J. Siemens, Phys. Lett. B126, 9 (1983).

[3] S. Ayik, M. Colonna and Ph. Chomaz, Phys. Lett. B353, 417 (1995).

[4] B. Jacquot et al., Phys. Lett. B 383 (1996) 247.

[5] J. Pouthas et al., (INDRA coll.), Nucl. Instr. and Meth. A 357 (1995) 418.

[6] M.F. Rivet et al. (INDRA coll.), Phys. Lett. B 430 (1998) 217.

[7] J.D. Frankland et al., (INDRA coll.) nucl-ex/0007019 and Nucl. Phys. A in press.

[8] J. F. Lecolley et al., Phys. Lett. B387 (1996) 460.
[9] L. Beaulieu et al., Phys. Rev. Lett. 77 (1996) 462.

[10] L. G. Moretto et al., Phys. Rev. Lett. 77 (1996) 2634.

[11] J. Randrup and B. Remaud, Nucl. Phys. A514 (1990) 339.

[12] Ph. Chomaz, G. F. Burgio and J. Randrup, Phys. Lett. B254 (1991) 340; G. F. Burgio, Ph. Chomaz and J. Randrup, Nucl. Phys A529 (1991) 157.

[13] Ph. Chomaz et al., Phys. Rev. Lett. 73 (1994) 3512.

[14] A. Guarnera et al., Phys.Lett B403 (1997) 191.

[15] J.D. Frankland et al., (INDRA coll.) nucl-ex/0007020 and Nucl. Phys A in press.

[16] L. Zamick, Phys. Lett. B45 (1973) 313.

[17] A. Guarnera, M. Colonna and Ph. Chomaz, Phys. Lett. B373 (1996) 267.

[18] M. Colonna, Nucl. Phys. A630 (1998) 136c.

[19] G. F. Bertsch, Z. Phys. A 289 (1978) 103.

[20] A. D. N'guyen, Ph. D. thesis, Université de Caen (1998), LPCC T 98-02 (1998).

[21] J. Bondorf et al., Phys. Rep. 257 (1995) 133.

[22] N. Le Neindre, Ph. D. thesis, Université de Caen, LPCC T 99-02 (1999).

[23] B. Bouriquet, G. Tăbăcaru et al., (INDRA coll.), to be published.

[24] L. Beaulieu et al., Phys. Rev. Lett. 84 (2000) 5971.

[25] H.S. Xu et al., Phys. Rev. Lett. 85 (2000) 716. 